\title{
ERRATUM
}

\section{Adherence to a healthy Nordic food index is associated with a lower incidence of colorectal cancer in women: the Diet, Cancer and Health cohort study - ERRATUM}

Cecilie Kyrø, Guri Skeie, Steffen Loft, Kim Overvad, Jane Christensen, Anne Tjønneland and Anja Olsen

(First published online 11 November 2013)

doi:10.1017/S0007114512002085, Published by Cambridge University Press, 3 July 2012.

We regret to announce that there Table 2 was published incorrectly in the article ${ }^{(1)}$.

All the data was correct, but the energy intake column was not rendered correctly. The publishers apologise for this error.

\section{Reference}

1. Kyrø C, Skeie G, Loft S, et al. (2013) Adherence to a healthy Nordic food index is associated with a lower incidence of colorectal cancer in women: the Diet, Cancer and Health cohort study. Br J Nutr 109, 920-927. Published by Cambridge University Press, 3 July 2012, doi:10.1017/S0007114512002085. 
Table 2. Baseline characteristics of all participants in the Diet, Cancer and Health cohort and by healthy Nordic food index scores of $0-1$ points (poorest adherence), 2-3 points and 4-6 points (best adherence)

(Medians, number of participants, percentiles and percentages)

\begin{tabular}{|c|c|c|c|c|c|c|c|c|c|c|c|c|c|c|c|c|}
\hline \multirow[b]{4}{*}{ Characteristic } & \multicolumn{16}{|c|}{ Food index score } \\
\hline & \multicolumn{8}{|c|}{ Men } & \multicolumn{8}{|c|}{ Women } \\
\hline & \multicolumn{2}{|r|}{ All } & \multicolumn{2}{|r|}{$0-1$} & \multicolumn{2}{|r|}{$2-3$} & \multicolumn{2}{|r|}{$4-6$} & \multicolumn{2}{|r|}{ All } & \multicolumn{2}{|r|}{$0-1$} & \multicolumn{2}{|r|}{$2-3$} & \multicolumn{2}{|r|}{$4-6$} \\
\hline & Median & P5-P95 & Median & P5-P95 & Median & P5-P95 & Median & P5-P95 & Median & P5-P95 & Median & P5-P95 & Median & P5-P95 & Median & P5-P95 \\
\hline$n$ & \multicolumn{2}{|r|}{26664} & \multicolumn{2}{|r|}{6608} & & 11966 & \multicolumn{2}{|r|}{8090} & \multicolumn{2}{|r|}{29216} & \multicolumn{2}{|r|}{5464} & \multicolumn{2}{|r|}{13728} & \multicolumn{2}{|r|}{10024} \\
\hline$\%$ & \multicolumn{2}{|c|}{100} & \multicolumn{2}{|r|}{25} & & 45 & \multicolumn{2}{|r|}{30} & & 100 & \multicolumn{2}{|r|}{19} & & 47 & \multicolumn{2}{|r|}{34} \\
\hline \multicolumn{17}{|l|}{ Cases } \\
\hline$n$ & \multirow{2}{*}{\multicolumn{2}{|c|}{$\begin{array}{l}567 \\
100\end{array}$}} & \multirow{2}{*}{\multicolumn{2}{|c|}{$\begin{array}{c}142 \\
25\end{array}$}} & & 273 & \multirow{2}{*}{\multicolumn{2}{|c|}{$\begin{array}{c}152 \\
27\end{array}$}} & & 458 & \multirow{2}{*}{\multicolumn{2}{|c|}{$\begin{array}{c}108 \\
24\end{array}$}} & & 203 & & 147 \\
\hline$\%$ & & & & & & 48 & & & & 100 & & & & 44 & & 32 \\
\hline Age (years) & 56 & $50-64$ & 55 & $50-64$ & 56 & $50-64$ & 56 & $50-64$ & 56 & $50-64$ & 55 & $50-64$ & 56 & $50-64$ & 56 & $50-64$ \\
\hline Schooling (\%) & & & & & & & & & & & & & & & & \\
\hline Short ( $\leq 7$ years) & & 35 & & 41 & & 35 & & 29 & & 31 & & 37 & & 32 & & 28 \\
\hline Medium ( $8-10$ years) & & 42 & & 42 & & 42 & & 40 & & 50 & & 49 & & 51 & & 50 \\
\hline Long ( $\geq 11$ years) & & 24 & & 17 & & 23 & & 31 & & 19 & & 15 & & 17 & & 22 \\
\hline Smoking status (\%) & & & & & & & & & & & & & & & & \\
\hline Never & & 26 & & 22 & & 25 & & 30 & & 44 & & 37 & & 43 & & 48 \\
\hline Past & & 35 & & 28 & & 35 & & 39 & & 24 & & 19 & & 23 & & 27 \\
\hline Current & & 40 & & 50 & & 40 & & 31 & & 33 & & 44 & & 35 & & 24 \\
\hline $\mathrm{BMI}\left(\mathrm{kg} / \mathrm{m}^{2}\right)$ & 26 & $21-33$ & 26 & $22-34$ & 26 & $22-33$ & 26 & $21-32$ & 25 & $20-34$ & 25 & $20-34$ & 25 & $20-34$ & 25 & $20-34$ \\
\hline $\begin{array}{l}\text { Waist } \\
\text { circumference }(\mathrm{cm})\end{array}$ & 95 & $81-114$ & 96 & $82-116$ & 95 & $82-113$ & 94 & $81-112$ & 80 & $67-103$ & 81 & $67-105$ & 80 & $67-103$ & 80 & $67-103$ \\
\hline Alcohol intake (g/d) & 19 & $2-80$ & 21 & $1-91$ & 19 & $2-78$ & 19 & $2-67$ & 9 & $0-42$ & 8 & $0-48$ & 10 & $0-43$ & 9 & $1-39$ \\
\hline Participate in sports (\%) & & 49 & & 38 & & 48 & & 58 & & 58 & & 45 & & 57 & & 68 \\
\hline $\begin{array}{l}\text { Intake of red and } \\
\text { processed meat }(\mathrm{g} / \mathrm{d})\end{array}$ & 139 & $67-254$ & 131 & $67-237$ & 140 & 67-252 & 144 & $65-272$ & 83 & $35-157$ & 78 & $35-145$ & 84 & $36-154$ & 87 & $34-166$ \\
\hline $\begin{array}{l}\text { Energy intake }(\mathrm{kJ} / \mathrm{d}) \\
\text { excluding alcohol }\end{array}$ & 9908 & $6414-14942$ & 8492 & $5518-12542$ & 9781 & $6666-14316$ & 11232 & $7898-16332$ & 8092 & $5092-12318$ & 6677 & $4159-10206$ & 7800 & $5216-11568$ & 9155 & $6241-13411$ \\
\hline
\end{tabular}

P5, 5th percentile; P95, 95th percentile. 